$\frac{224}{11-5-0}=$

Or. 1824

\title{
Model for Ground Motion and Atmospheric Overpressure Due to Underground Nuclear Explosion
}

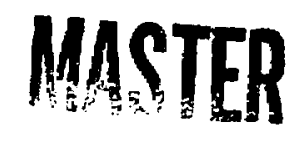

范

$\geqslant$

京 
MODEL FOR GROUND MOTION AND ATMOSPHERIC OVERPRESSURE

DUE TO UNDERGROUND NUCLEAR EXPLOSION

by

Huan Lee and James J. Walker

\section{ABSTRACT}

A physical model is proposed to describe the ground motion pattern resulting from an underground nuclear explosion in an idealized homogeneous medium. Irregular behaviors in the observed ground motion are assumed to be perturbations caused by the local inhomogeneity of the ground medium. Our model correlates the ground motions at any point in the spalled zone to the initial acceleration pulse at the ground zero. Interestingly, the model predicts that the ground motion first comes to a stop at a definite radius about the ground zern, and the region expands both outward and inward as time goes on. We belfeve that this result is closely related to a phenomenon observed at NTS. In the far field approximation, we also calculate the overpressure in the lower atmosphere generated by the ground motion. We demonstrate that the irregular component of the ground motion does not affect the overpressure history in any significant way. Consequently the model ground motion can be used as a good approximation in generating the atmospheric overpressure.

I. INTRODUCTION

The ground motion (GM) associated with an underground nuclear explosion is thought to be powerful enough to generate an acoustic wave in the atmosphere. The pattern of the GM, as measured by accelerometers, varies with 
the yield of the device, the depth of burial, the distance away from ground zero, and, finally, the type and the composition of the ground medium. Loosely speaking, the GM can be said to have two components, one regular and one irregular. The regular component has a time and spatial correlation which can presumably be understood by physical considerations of a homogeneous ground medium. The irregular component, which is the perturbation resulting from the local inhomogeneity of the ground medium, evidently is quite random in nature. The irregular component causes the apparent complexity exhibited in the measured GM pattern. Fortunately for us, it is the regular component that plays a dominating role in generating the characteristic pulse shape of the overpressure in the air. In this report we present a physical model for the regular component of the GM. The model provides us a coherent picture of how the GM pattern depends on various parameters. Furthemore, it possesses the essential st"uctures so that an approximate overpressure pulse can be simulated in the lower atmosphere.

In the next section, we first discuss the physical considerations which form the basis for modeling the GM. We formulate the GM in terms of a timeand position-dependent acceleration function, which can be specified completely if the initial acceleration pulse at ground zero is known. In Section III, we analyze when the GM comes to a stop as a function of distance and time. We arrive at an interesting conclusion that it occurs first at a definite radius about the ground zero, and the region subsequently expands both inward and outward. The result is used to explain a phenomenon observed in several NTS events. In Section IV, we first calculate the atmospheric overpressure generated by a $\delta$-function acceleration pulse, in the far field approximation. The result is compared with the one obtained in the piston model. We then proceed to calculate the overpressure due to a general acceleration pulse coming from a point source. We discuss why the irregular component of the $G M$ does not affect the overpressure in a significant way, thus establish that the model GM can be a good approximation in generating the overpressure in the air. 


\section{MODEL FOR GROUND MOTION}

To consider the regular component of the GM, our starting point is to assume that the ground medium is homogeneous. It follows that the shock front is spherical and the $G M$ is therefore circularly symetric about the ground zero. Since only the vertical component of the ground acceleration contributes to the atmospheric overpressure, it is sufficient for our purpose to describe the $G M$ by the vertical acceleration as a function of time and location. Simply put, our model for the GM consists of a sharp initial acceleration pulse (IAP), followed by a period of free fall, finally ended with another sharp upward acceleration pulse. $A_{3}$ the subsequent discussion will show, the model enables us to specify the acceleration history at any point once the IAP at ground zero is known.

The IAP starts at the arrival of the ground shock originated from the explosion center. We assume that the duration of the IAP does not vary with the slant distance (SD). In the elastic zone this is indeed the case. The amplitude of the IAP, however, is expected to scale down as the SD increases. In the absence of energy loss, we know that the amplitude of a spherical pressure pulse should be inversely proportional to the radius. One can argue as follows: Let $\Gamma$ be the width of the acceleration pulse, then $c \Gamma$ is the thickness of the spherical shell that is set in motion, where $c$ is the sound velocity. The energy carried by the pulse is then $\propto 4 \pi r^{2}(c \Gamma)_{\rho} v^{2}$, where $\rho$ is the density, $v$ the peak velocity, and $r$ the propagation distance. When $T$ remains fixed, the amplitude of the acceleration pulse is proportional to the peak velocity, hence varies as $1 / r$. In reality, the energy carried by the pressure pulse does diminish as it propagates in a realistic medium. The inelastic effects also tend to make the pulse width broader as it travels out. This means in general that the amplitude of the acceleration pulse scales down as $1 / r$ or faster. ${ }^{1}$ Furthermore, since only the vertical component contributes to the overpressure, projecting in the vertical direction adds another inverse power ${ }^{2}$ dependence on the SD.

In the spalled zone, the top surface layer, once spalled by the IAP, will be in a free fall state. The duration of the free fall motion at a given SD is in principle related to the amplitude of the IAP. In an idealized picture, the free fall would end when the spallina layer returns to its original position. A sharp acceleration pulse will result from the impact. Since the 
elasticity of the surface soil or rock is quite low, no significant bouncing will occur after the collision. The collision time is so short that practically we can approximate the second acceleration pulse by a $\delta$-function. The ccefficient of the $\delta$-function is fixed by the condition that the time integral of the pulse should be (nearly) equal to the impact speed.

Following the above discussion, we are now in a position to specify the ground acceleration as a function of time $t$ and distance $r$, denoted by $A(t, r)$. It is more convenient to define $A(t, r)$ through a function $B(t, r)$ in which the time variable is retarded by the propagation time of the ground shock, i.e.,

$$
A(t, r)=B\left(t-t_{g}(r), r\right)
$$

where

$$
t_{g}(r)=\left(b^{2}+r^{2}\right)^{1 / 2} / c_{g},
$$

and

$$
\begin{aligned}
& b \quad=\text { depth of burial of the source, } \\
& c_{g} \quad=\text { velocity of sound in ground. }
\end{aligned}
$$

A schematic drawing to illustrate the geometry is given in Fig. 1.

The function $B(t, r)$ is then defined as ${ }^{3}$

$$
\begin{array}{ll}
B(t, r)=0 & t<0, \\
B(t, r)=S(r) A(t) & 0<t<\alpha, \\
B(t, r)=-g & a<t<B(r), \\
B(t, r)=V(r) \delta(t-B(r)) & t>B(r),
\end{array}
$$

where $g$ is the gravitational acceleration, and $S(r)$ is the SD scaling function, ${ }^{1}$ namely

$$
s(r)=\left(1+r^{2} / b^{2}\right)^{-n / 2}, n \geq 2 \text {. }
$$




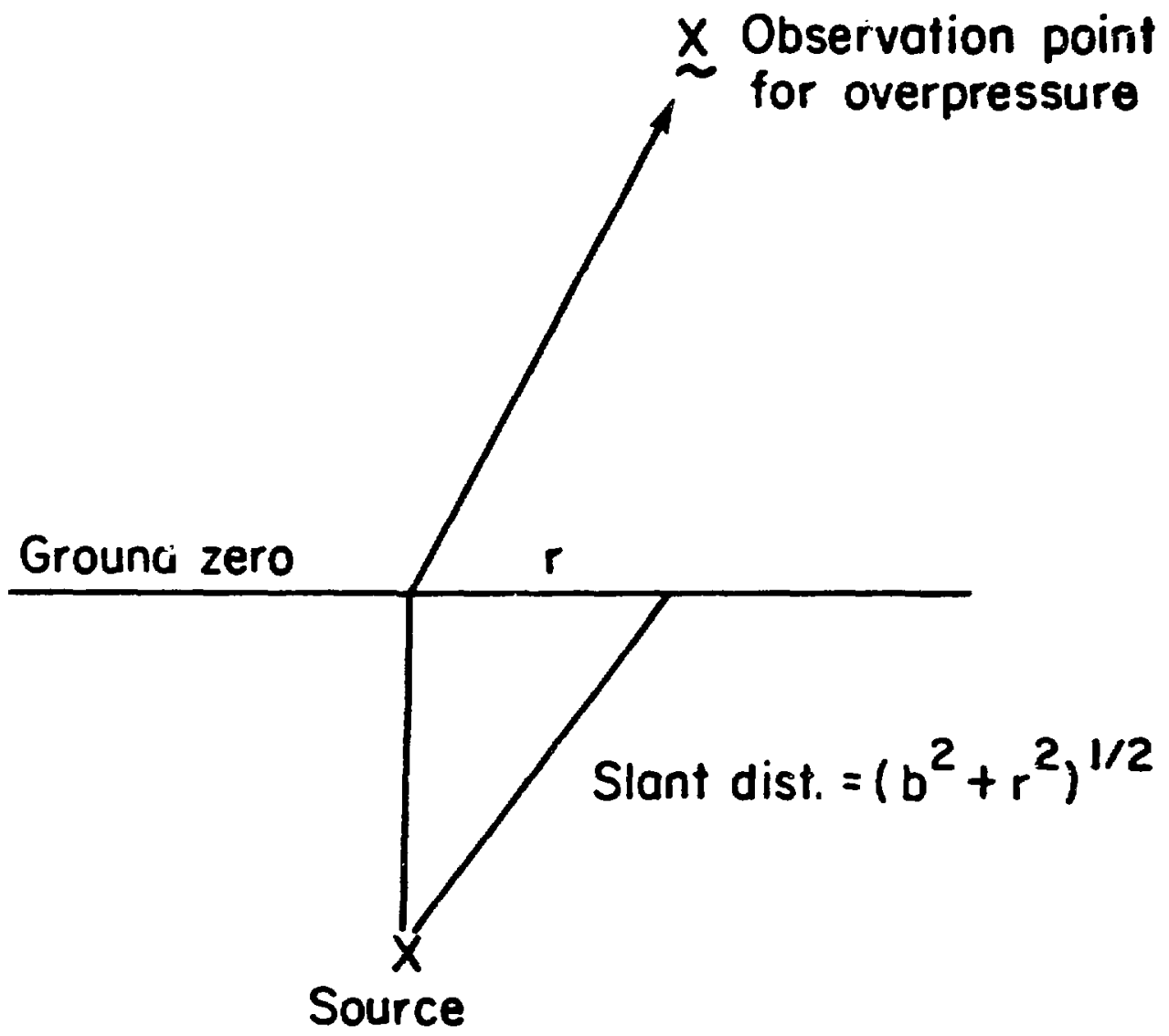

Fig. 1. The schematic geometry describing ground motion. 
$A(t)$ is the acceleration function of the IA at ground zero. The free fall duration $B(r)-\alpha$ and the impact speed $V(r)$ can be derived from $S(r)$ and $A(t)$. With $B(t, r)$ specified, we can calculate the time-shifted velocity function $V(t, r)$ and displacement function $D(t, r)$ as

$$
V(t, r)=\int_{0}^{t} B\left(t^{\prime}, r\right) d t^{\prime}
$$

and

$$
D(t, r)=\int_{0}^{t} V\left(t^{\prime}, r\right) d t^{\prime}
$$

From our earlier discussion $B(r)$ should satisfy the condition

$$
D(B(r), r)=0 \text {, }
$$

and the impact speed is just

$$
V(r)=-V(B(r), r)
$$

To solve for $\beta(r)$ and $V(r)$, we notice that in the time interval between $\alpha$ and $B(r)$ the motion is free fall. Therefore, we have the relations

$$
V(r)+V(\alpha, r)=g[\beta(r)-\alpha]
$$

and

$$
\begin{aligned}
v^{2}(r)-v^{2}(\alpha, r) & =2 g[D(\alpha, r)-D(B(r), r)] \\
& =2 g D(\alpha, r) .
\end{aligned}
$$

These lead to

$$
V(r)=\left[v^{2}(\alpha, r)+2 g D(\alpha, r)\right]^{1 / 2}
$$


and

$$
B(r)=\alpha+1 / g[V(\alpha, r)+V(r)] .
$$

Thus both $V(r)$ and $\beta(r)$ can be expressed in terms of $V(\alpha, r)$ and $D(\alpha, r)$. So $A(r, t)$ is completely determined by the IAP at ground zero.

If the IAP $A(t)$ is symmetric about its peak, it is straightforward to show that $V(\alpha, r)$ and $D(\alpha, r)$ are further related by

$$
D(\alpha, r)=1 / 2 \alpha \gamma(\alpha, r)
$$

Equation (11) can be written as

$$
V(r)=V(\alpha, r)[1+g \alpha / V(\alpha, r)]^{1 / 2}
$$

Experimentally the observed initial acceleration pulses are approximately symmetric about their peaks, so the relation in Eq. (13) actually holds very well for the measured data. It should be stressed that Eq. (14), together with Eq. (12), imply that the GM after the IAP can be completely specified by the peak velocity $V(\alpha, r)$ alone, and is iridependent of the shape of the IAP. The significance of this observation will be discussed in Section IV.

Having presented the idealized description of the GM, we would like to compare it with the real-world observation. We plot a typical acceleration history in Fig. $2(a)$ and the corresponding velocity history in Fig. 2(b). Their typical observed counterparts are shown in Fig. 3(a) and Fig. 3(b). Generally speaking, the measured GM exhibits a sharp IAP, followed by an identifiable period of free fall which usually ends with a pronounced acceleration pulse. The duration of the IAP normally remains fairly constant as the slant distance changes. In addition to these regular features, the observed GM displays the irregularity that at one point the free fall is uninterrupted while at another it may be interrupted by one or two (occasionally more) very narrow acceleration pulses, presumably due to collisions among the multi-spalled ground layers. Whenever the interruption is absent, we find, not surprisingly, that the length of the free fall period agrees quite well with the value $B(r)-\alpha$ calculated by Eq. (12). In case there are interruptions such as shown in Fig. 3(a), the duration of free fall tends to be ionger than calculated, but not much. 


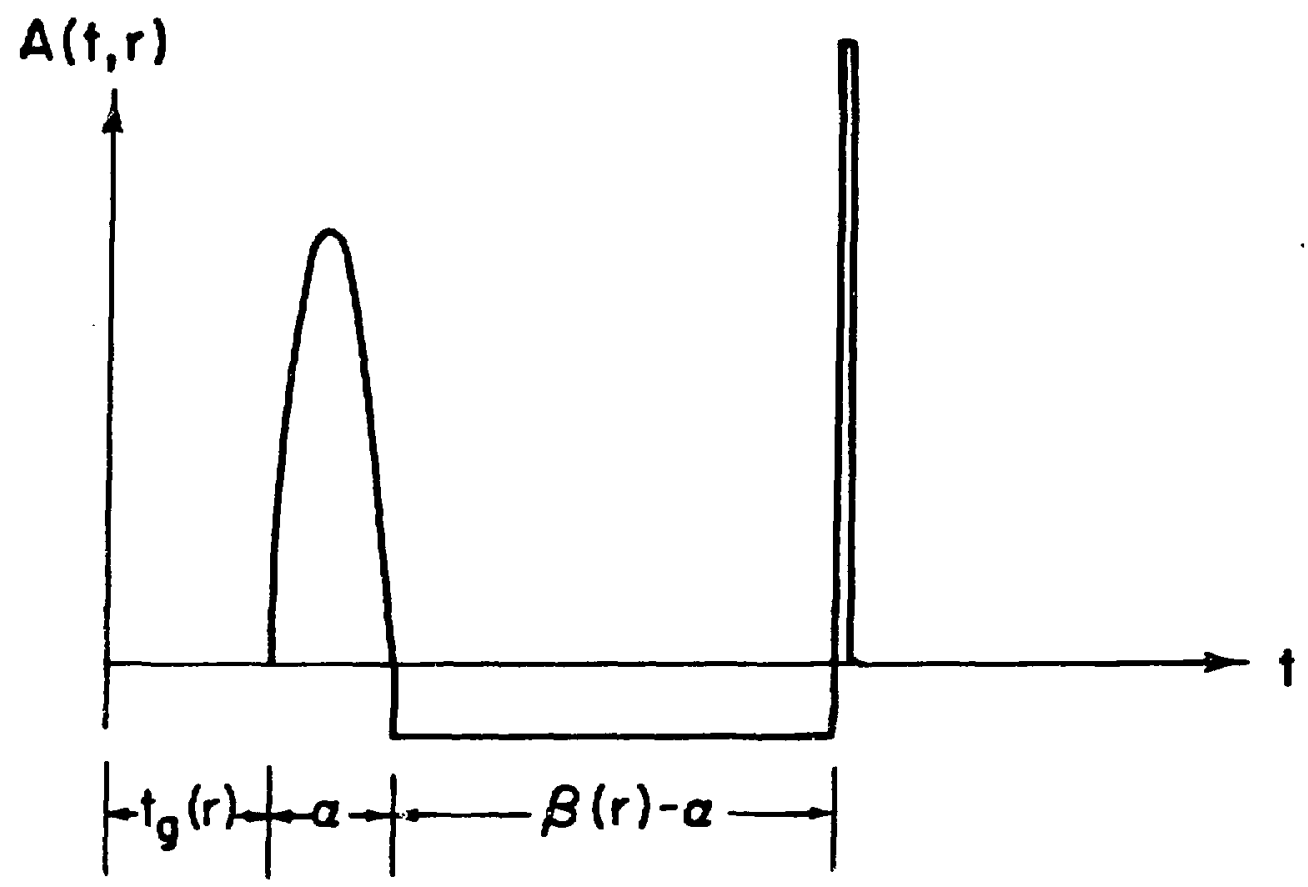

Fig. $2(a)$. Model pattern of ground acceleration.

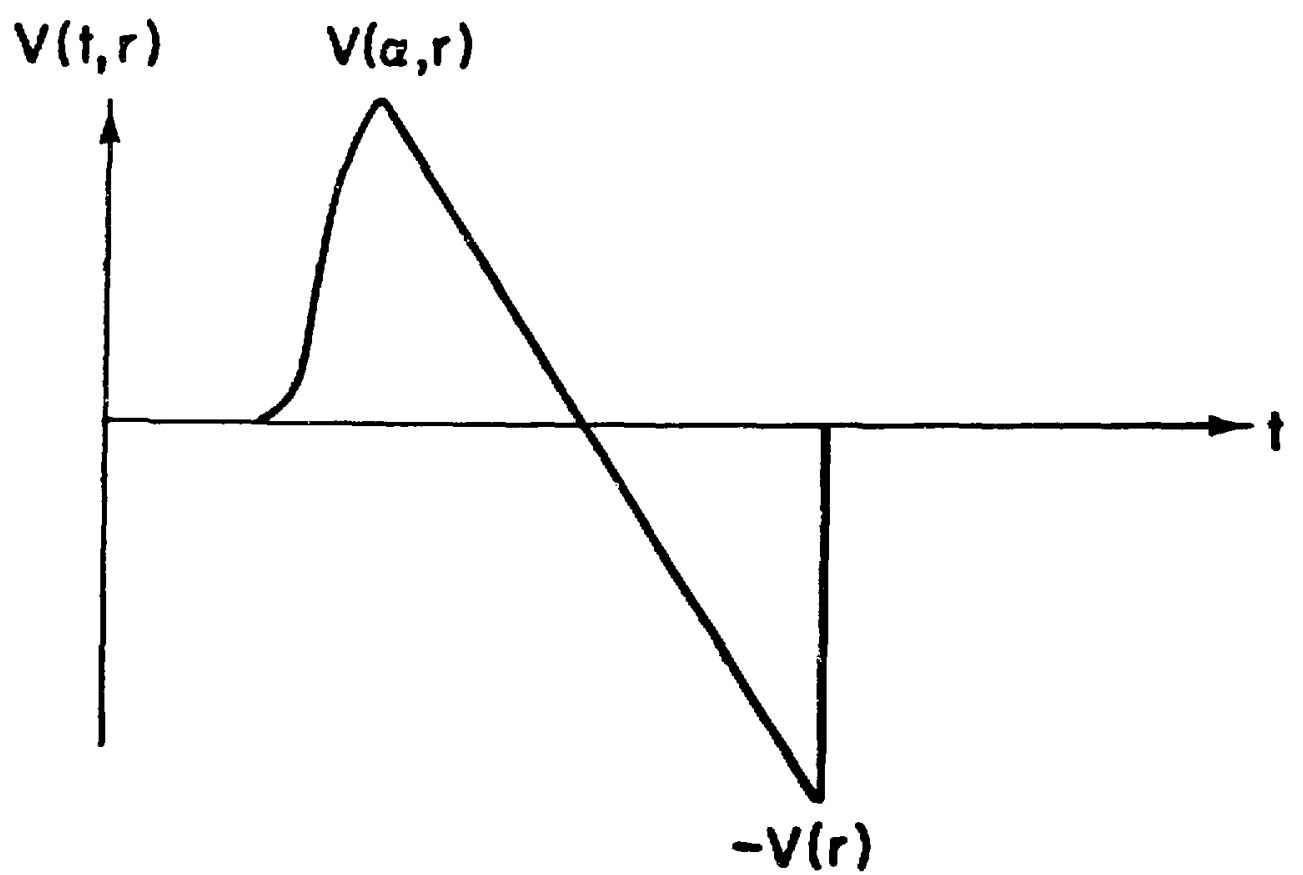

Fig. 2(b). Model pattern of ground velocity. 


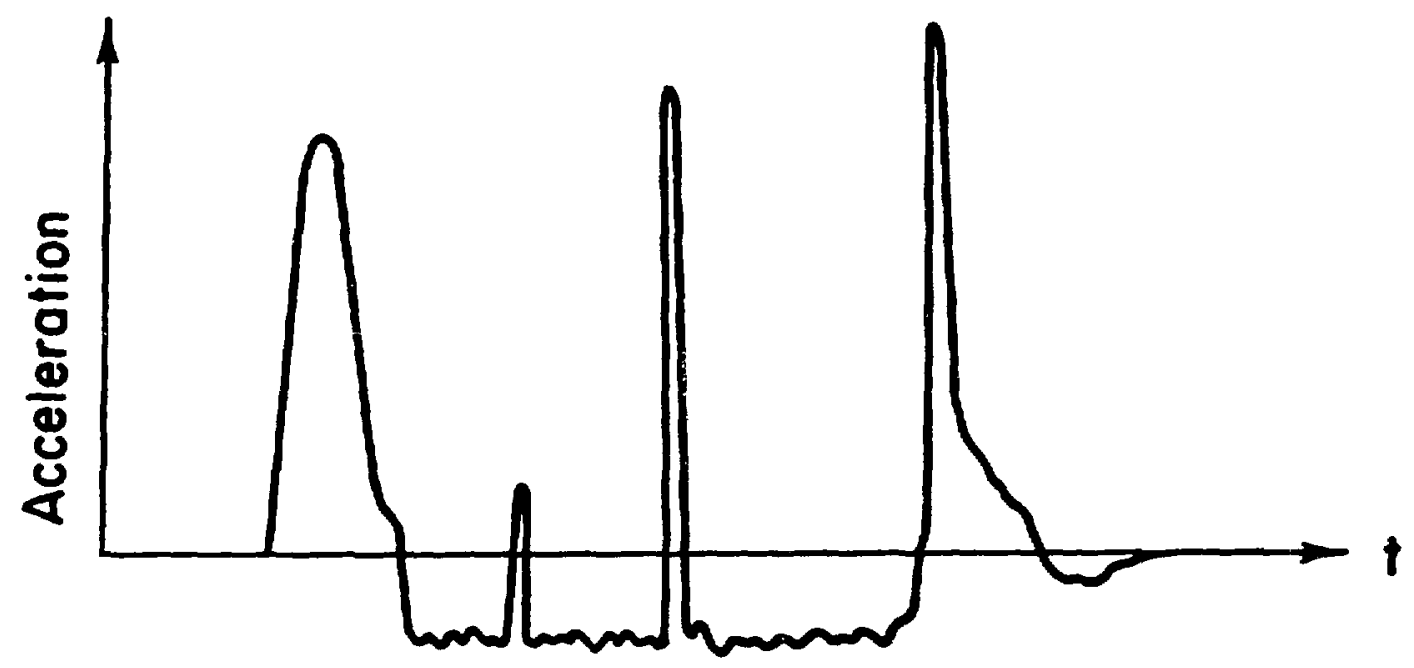

Fig. 3(a). A typical measured ground acceleration.

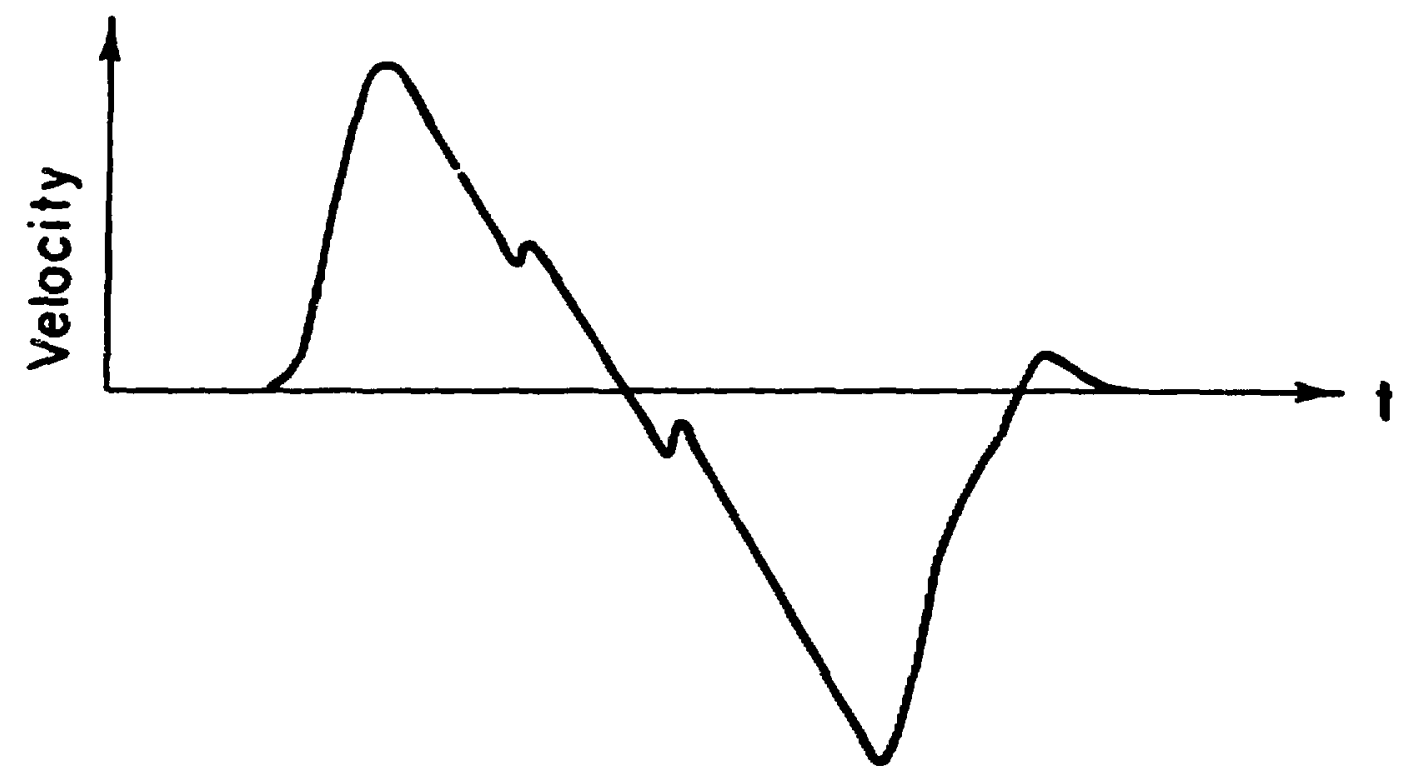

Fig. 3(b). A typical measured ground velocity. 
Another point worth mentioning about the observed $G M$ is that the acceleration pulse at the end of the free fall generally has a damped oscillating tail such as shown in Fig. 3(a). Although the acceleration patterns shown in Figs. $2(a)$ and $3(a)$ seem to have marked differences, their velocity patterns as displayed in Figs. $2(b)$ and $3(b)$ are quite similar. It will become clear in Section IV that the velocity pattern has a closer semblance to the overpressure than the acceleration pattern itself. This is why we claim that the model, which includes only the regular component of the GM, can be a good approximation to the real data in generating the overpressure.

As far as we can tell, the interrupting acceleration pulses which occur in the measured GM do not exhibit any systematic spatial or temporal correlations. For the purpose of calculating the overpressure generated by the $G M$, the net effect of these interrupting pulses contributed from the entire spalled surface is equivalent, in an average sense, to having a lower $g$ value during free fall. So the model can be "fine-tuned" simply by using an "effective" $g$ value if so desired.

III. GROUND MOTION PHENOMENOLOGY

From their explicit expressions

$$
V(\alpha, r)=S(r) \int_{0}^{\alpha} A(t) d t
$$

and

$$
D(\alpha, r)=S(r) \int_{0}^{\alpha} d t \int_{0}^{t} A\left(t^{\prime}\right) d t^{\prime}
$$

we see that $V(\alpha, r)$ and $D(\alpha, r)$ are linear in the scaling function $S(r)$. This implies that the duration of the free $f a l l, B(r)-\alpha$, decreases as $r$ increases. As it turns out, within a certain radius, the decreasing rate of $\beta(r)$ - $\alpha$ is faster than the increasing rate of $t_{g}(r)$, as $r$ is increasing. This leads to the interesting implication that even though the initial shock 
arrives later in time for larger $r$, the GM could stop sooner. Let us elaborate this point by the following analysis:

Let $t(r)$ be the slap-down time (i.e., when the free fall ends) at a point $r$; we have

$$
t(r)=t_{g}(r)+\beta(r)
$$

As shown in the Appendix, we can derive

$$
\frac{d t(r)}{d r}=\frac{r}{\left(r^{2}+b^{2}\right)}\left[t_{g}(r)-\frac{n(V(\alpha, r)+V(r))^{2}}{2 g V(r)}\right] \text {. }
$$

Since $t_{g}(r)$ is monotonically increasing whereas $[V(\alpha, r)+V(r)]^{2} / V(r)$ is monotonically decreasing with $r$, it is evident that

$$
\frac{d t(r)}{d r} \gtrless 0 \text { for } r \gtrless R \text {, }
$$

where $R$ is the solution of $r$ for

$$
\frac{d t(r)}{d r}=0
$$

Equation (19) implies that the GM will stop first at a fixed radius $R$. Inside $R$, the slap-down process rapidly moves inward, whereas outside $R$, the opposite is true. Experimentally we have indeed observed the following phenomenon in the aerial iV pictures taken over many NTS events. A couple of seconds after the zero time, there would appear a dark ring (sometimes irregular in shape) rapidly shrinking in toward ground zero. It is compelling to relate this phenomenon to the conclusion implied by Eq. (19). The observed initial ring size and rapidity with wich the ring shrinks are in fair agreement with the calculated values.

Numerical solution is needed to obtain an accurate value of $R$. However, it is informative to take a look at the approximate analytic solution in Eq. (A-7) derived in the Appendix, 


$$
R \approx b\left\{\left[5 n V(\alpha, 0) / 2 g t_{g}(0)\right]^{2 /(n+1)}-1\right\}^{1 / 2} \text {. }
$$

Normally the depth of burial, $D$, is chosen to be proportional to $\gamma^{1 / 3}$, where $Y$ is the yield of the device. Consequently, we have $t_{g}(0) \propto y^{1 / 3}$. The peak velocity at ground zero, $V(\alpha, 0)$, in general depends on $Y$ as well as the ground medium. For a given type of medium, however, one can argue from energy consideration that $V(\alpha, 0)$ is essentially independent of $Y$ provided $b \propto \gamma^{1 / 3}$. in this case, the square root quantity in Eq. $(A-6)$ is rather insensitive to the variation of $Y$, and we conclude that $R \propto b \propto Y^{1 / 3}$. In passing, we like to stress that the type of ground medium can affect $V(\alpha, 0)$ substantially and also the value of scaling power $n$.

IV. ATMOSPHERIC OVERPRESSURE.

Let $A(t, r)$ be the function describing the vertical ground acceleration, in the far field approximation the overpressure at a given point $x$ and time $t$ is given by 4

$$
P(\underset{\sim}{x}, t)=\frac{\rho}{2 \pi} \int A\left(t-t_{a}, \underset{\sim}{r}\right)|\underset{\sim}{x}-\underset{\sim}{r}|^{-1} d^{2} \underset{\sim}{r},
$$

where $\rho$ is the air density and $t_{a}=|\underset{\sim}{x}-\underset{\sim}{r}| / c$ with $c$ being the sound velocity in air. In particular, if $A(t, r)$ is circularly symmetric with respect to the ground zero, and $\underset{\sim}{x}$ is vertically above the latter, then Eq. (21) can be reduced to a one-dimensional integral. Let $\underset{\sim}{x}=(0,0, h)$ in a coordinate system with the ground zero being chosen as the origin, we have

$$
P(n, t)=\rho \int_{0}^{\infty} A\left(t-t_{a}(r), r\right)\left(n^{2}+r^{2}\right)^{-1 / 2} r d r \text {, }
$$

where $t_{a}(r)=\left(h^{2}+r^{2}\right)^{1 / 2} / c$. Many essential features of the overpressure generated by the $G M$ can be learned by studying the above integral. In the following, our discussion will be based entirely or it. 
It is worthwhile to mention at this point that, if the acceleration function $A(t, r)$ is identical and synchronous everywhere within a radius $r=$ $r_{0}$, then the overpressure is simply the velocity integra1.5 In this "piston" model, we can write

$$
A(t, r)=A(t)
$$

and

$$
\begin{aligned}
P(n, t) & =\rho \int_{0}^{r_{0}} A\left(t-t_{a}(r)\right)\left(h^{2}+r^{2}\right)^{-1 / 2} r d r \\
& =\rho c \int_{t_{a}(0)}^{t_{a}\left(r_{0}\right)} A\left(t-t_{a}(r)\right) d t_{a}(r) \\
& =\rho c \int_{t-t_{a}(0)}^{t-t_{a}\left(r_{0}\right)} A\left(t^{\prime}\right) d t^{\prime} \\
& =\rho c\left[V\left(t-t_{a}(0)\right)-V\left(t-t_{a}\left(r_{0}\right)\right)\right]
\end{aligned}
$$

The last equality stated that $P(h, t)$ is the difference between the velocities at retarded time $t-t_{a}(0)$ and $t-t_{a}\left(r_{0}\right)$. For large enough $r_{0}$ so that $V\left(t-t_{a}\left(r_{0}\right)\right)$ is zero for all practical $t$ values of interest, the overpressure is simply proportional to velocity function $v\left(t-t_{a}(0)\right)$.

In particular, if $A(t)$ is a $\delta$-function, then $P(h, t)$ is simply a square pulse with a non-zero value between $t-t_{a}(0)$ and $t-t_{a}\left(r_{0}\right)$ and $P(h, t)$ becomes a step function for $r_{0}+\infty$.

It is useful and illuminating to work out first the overpressure generated by a d-function ground acceleration due to a point source underground. Since the shock will arrive at the surface at different times for different: SD, we write the acceleration function as

$$
A(r, t)=\delta\left(t-t_{g}(r)\right) S(r),
$$


where $t_{g}(r)$ is defined in Eq. (2) and $S(r)$ is the scaling function. Using Eq. (22), we have

$$
q_{\delta}(h, t)=\rho \int_{0}^{r_{0}} \delta\left(t-t_{g}(r)-t_{a}(r)\right) s(r)\left(h^{2}+r^{2}\right)^{-1 / 2} r d r .
$$

We can rewrite

$$
\delta\left(t-t_{g}(r)-t_{a}(r)\right)=\delta\left(r^{2}-x(t)\right)\left|\frac{\partial t_{g}}{\partial r^{2}}+\frac{\partial t_{a}}{\partial r^{2}}\right| r^{2}=x(t),
$$

where $x(t)$ is the solution of the equation

$$
t \cdot\left(h^{2}+x\right)^{1 / 2} / c-\left(b^{2}+x\right)^{1 / 2} / c=0 .
$$

It is straightforward to solve the above equation and find

$$
x(t)=\left\{\frac{2 t^{2}}{c^{2}}-\left(\frac{h^{2}}{c^{2}}-\frac{b^{2}}{c_{g}^{2}}+t^{2}\right) Q-\frac{2 t}{c}\left[Q\left(\frac{b^{2}-h^{2}}{c_{g}{ }^{2}}-t^{2}\right)+\frac{t^{2}}{c^{2}}\right]^{1 / 2}\right\} Q^{-1},
$$

where $Q=\frac{1}{c^{2}}-\frac{1}{c_{g}^{2}}$.

Substituting (26) into (25) and integrating over $r^{2}$, we obtain

$$
P_{\delta}(h, t)={ }_{\rho} c c g_{g} S(\sqrt{x})\left(b^{2}+x\right)^{1 / 2} /\left[c g_{g}\left(b^{2}+x\right)^{1 / 2}+c\left(h^{2}+x\right)^{1 / 2}\right] \text {, for } t_{1}<t<t_{2} \text {, }
$$

and

$$
P_{\delta}(h, t)=0 \text { otherwise. }
$$


Where

$$
t_{1}=\frac{h}{c}+\frac{b}{c}
$$

and

$$
t_{2}=\left(h^{2}+r_{0}^{2}\right)^{1 / 2} / c+\left(b^{2}+r_{0}^{2}\right)^{1 / 2} / c_{g} .
$$

We note that the propagation time of the wave aiong different paths causes a smearing of the $\delta$-function acceleration through r-integration. Thus, the overpressure is nonvanishing over a finite period determined by $r_{0}$. Since $x(t)$ increases with $t$, so $P_{\delta}(h, t)$ is monotonically decreasing with $i$ between $t_{1}$ and $t_{2}$. Compared with the overpressure resulting from a synchronous $\delta-$ function acceleration, we have two factors contributing to the difference. The scaling function, $s(r)$, is the one most responsible for the decreasing of $P_{\delta}(h, t)$ with $t$. The other factor, $\left(b^{2}+x\right)^{1 / 2} /\left[c_{g}\left(b^{2}+x\right)^{1 / 2}+c\left(h^{2}+x\right)^{1 / 2}\right]$, decreases with increasing $t$ very slightly. In the limit $b+\infty$, the result of Eq. (28) approaches the one in the piston model, as it should.

After working out $P_{\delta}(h, t)$, we can easily obtain the overpressure due to an acceleration pulse of the general form

$$
A(r, t)=A\left(t-t_{g}(r)\right) S(r)
$$

Without loss of generality, the function $A(x)$ can be assumed to have a finite support in $[0, \Delta]$. We first put $A(r, t)$ into a representation

$$
A(r, t)=S(r) \int_{0}^{\Delta} A(T) \delta\left(t-T-t_{g}(r)\right) d T .
$$


It foilows that

$$
\begin{aligned}
P(h, t) & =\rho \int_{0}^{r} O_{A}\left(r, t-t_{g}(r)\right)\left(h^{2}+r^{2}\right)^{-1 / 2} r d r \\
& =\rho \int_{0}^{\Delta} A(T) d T \int_{0}^{r} O_{S}(r) \delta\left(t-T-t_{a}(r)-t_{g}(r)\right)\left(h^{2}+r^{2}\right)^{-1 / 2} r d r \\
& =\int_{0}^{\Delta} A(T) P_{\delta}(h, t-T) d T .
\end{aligned}
$$

If the puise $A(T)$ is such that $\Delta \ll\left(t_{2}-t_{1}\right)$, it is easy to see from the convolution integral above what is the general shape of $P(h, t)$. For $t_{1}<t<$ $t_{1}+\Delta$, it rises from zero to the maximum steeply; for $t_{1}+\Delta<t<t_{2}$, it decreases monctonically just like $P_{\delta}(h, t)$; and, finally, for $t_{2}<t<t_{2}+\Delta$, it drops off steeply to zero.

We have discussed in Section II that the model GM provides a close representation of the actual one in terms of velocity history. As implied most clearly in the piston model, the overpressure history is simply proportiona? to the velocity hiscory of the GM. In the general case, this reiationship still holds approximately. This fact supports our earlier assertion that the model GM can be a good approximation to the real data in generating the atmospheric overpressure. 


\section{APPENDIX}

To derive the Eq. (18), we use the fact that both $V(\alpha, r)$ and $D(\alpha, r)$ are linear in $S(r)$, therefore

$$
\begin{aligned}
\frac{d V^{\sigma}(\alpha, r)}{d r} & =\frac{d s}{d r} \frac{\partial V^{\sigma}(a, r)}{\partial S} \\
& =-\frac{n r S}{r^{2}+b^{2}} \frac{\partial V^{\sigma}(\alpha, r)}{\partial S} \\
& =-\frac{n r \sigma}{\left(r^{2}+b^{2}\right)} V^{\sigma}(\alpha, r) .
\end{aligned}
$$

and, similarly,

$$
\frac{d D(\alpha, r)}{d r}=-\frac{n r}{\left(r^{2}+b^{2}\right)} D(\alpha, r)
$$

Using Eqs. (A-1), (A-2), and (17), we have

$$
\begin{aligned}
\frac{d t(r)}{d r} & =\frac{d t_{g}(r)}{d r}+\frac{1}{g}\left(\frac{d v(\alpha, r)}{d r}+\frac{d V(r)}{d r}\right) \\
& =\frac{r}{c_{g}}\left(r^{2}+b^{2}\right)^{-1 / 2}-\frac{n r}{g\left(r^{2}+b^{2}\right)}\left[V(\alpha, r)+\frac{v^{2}(\alpha, r)+g D(\alpha, r)}{V(r)}\right] \\
& =\frac{r}{\left(r^{2}+b^{2}\right)}\left[t_{g}(r)-\frac{n[V(\alpha, r)+V(r)]^{2}}{2 g V(r)}\right] .
\end{aligned}
$$

We have used Eq. (11) in obtaining the last step of Eq. (A-3).

To obtain an approximate analytic solution for the equation $d t(r) / d r=0$, we use the fact that in a typical NTS event, $(\beta(0)-\alpha)$ is usually much larger than $\alpha$. Dividing Eq. (10) by Eq. (9) and using Eq. (13), we get

$$
V(0)-V(\alpha, 0)=\alpha V(\alpha, 0) /(\beta(0)-\alpha) \text {, }
$$


which implies $V(0)$ is just slightly larger than $V(a, 0)$. Therefore, we can approximately write

$$
\frac{V(r)}{V(a, r)}-\sqrt{S(r)}=\left(1+r^{2} / b^{2}\right)^{n / 4}
$$

We note that, to within an accuracy of $20 \%$, we can set $(x+y)^{2} / x y=5$ for $1 / 4$ $<x / y<4$. Now if $V(R) / V(\alpha, R)<4$ is valid, a posterfori, we can approximate the equation $d t(R) / d R=0$ by

$$
t_{g}(R)=5 n V(\alpha, R) / 2 g
$$

This leads to

$$
R=b\left\{\left[5 n V(a, 0) / 2 g t_{g}(0)\right]^{2 /(n+1)} \cdots 1\right\}^{1 / 2},
$$

to within an accuracy about $20 /(n+1)$ per cent. With $R$ given by the above, we have

$$
\frac{V(R)}{V(\alpha, R)}=\left(1+r^{2} / b^{2}\right)^{n / 4}=\left[5 n V(\alpha, 0) / 2 g t_{g}(0)\right]^{n / 2(n+1)} \text {. }
$$

For all the NTS events for which we have GM data, the quantity $\left[5 \mathrm{nV}(\alpha, 0) / 2 \mathrm{gt}_{G}(0)\right]$ never exceeds 20 , or equivalently $V(R) / V(\alpha, R)<3.1$, even if we take $n=3$. Therefore, the approximation used in writing Eq. $(A-6)$ is justified a posteriori. 


\section{REFERENCES AND FOOTNOTES}

1. Sraling exponent in slant distance varies with the composition of the ground medium. A thorough investigation of this, based on experimental data, was done by W. R. Perret and R. C. Bass, Free-field Ground Motion Induced by Underground Expiosion, SAND7 4-0252 (February 1975).

2. This is, of course, an oversimplified statement. Detailed discussions concerning this aspect can be found in J. R. Banister, D. M. Ellett, C. R. Mehl, and F. F. Dean, Stresses and Strains Developed by the Reflection of Seismic Waves at a Free Surface, SAND78-2361 (March 1979).

3. After this work was completed, we were grateful to J. R. Banister for bringing to our attention a paper by $R$. $K$. Coie, Jr., Acoustical Calculation of Grourd-Shuck-Induced Air Blast, SC-RR-7u-734, (November 1970), in which a similar approach was made to calculate the atmospheric overpressure due to the ground acceleration. However, his GM model is different from ours. To model the GM, he was mainly motivated by the $\mathrm{N}$ wave shape of the observed overpressure data, rather than formulating it by physical considerations as done in this paper.

4. P. M. Morse and K. U. Ingard, Theoretical Acoustics (McGraw-Hil1, 1968).

5. The same conclusion was also obtained in Ref. 4. 